\title{
Periodicity Hub and Nested Spirals in the Phase Diagram of a Simple Resistive Circuit
}

\author{
Cristian Bonatto and Jason A. C. Gallas \\ Instituto de Física, Universidade Federal do Rio Grande do Sul, 91501-970 Porto Alegre, Brazil
}

(Received 14 April 2008; published 1 August 2008)

\begin{abstract}
We report the discovery of a remarkable "periodicity hub" inside the chaotic phase of an electronic circuit containing two diodes as a nonlinear resistance. The hub is a focal point from where an infinite hierarchy of nested spirals emanates. By suitably tuning two reactances simultaneously, both current and voltage may have their periodicity increased continuously without bound and without ever crossing the surrounding chaotic phase. Familiar period-adding current and voltage cascades are shown to be just restricted one-parameter slices of an exceptionally intricate and very regular onionlike parameter surface centered at the focal hub which organizes all the dynamics.
\end{abstract}

Bifurcations are familiar phenomena routinely used nowadays as sensitive indicators of drastic changes in dynamical behaviors in physics and related sciences [15]. In fact, the past two decades witnessed extensive efforts to describe how bifurcations between periodic and chaotic oscillations unfold in all sorts of systems. They are ubiquitous features supported by all theoretical descriptions, based on models ranging from "simple" discrete-time mappings to sophisticated continuous-time multidimensional flows ruled by sets of differential equations. Bifurcation phenomena involving the variation of just a single parameter, referred to as codimension-one bifurcations, are now reasonably well understood [1-5]. In contrast, fragmentary information is available about much more realistic situations requiring the simultaneous variation of at least two independent parameters (codimension two).

The quintessential example of a codimension-two bifurcation and a current hot topic of research involves homoclinic orbits in the vicinity of a saddle focus, trajectories biasymptotic to a nonhyperbolic stationary point [6-10]. In this setup Shilnikov proved a celebrated theorem stating that, at nearby parameter values, one finds trajectories belonging to multiple horseshoes and in correspondence with the full shift on $n$ symbols [6-8]. While it is well known that familiar codimension-one signatures may be seen locally in higher codimension, no globally encompassing description is available about the organization over large parameter ranges, particularly about how distinct bifurcation scenarios interconnect and influence each other. For flows, no codimension-two investigation of the structuring of the abundant chaotic phases seems to have been done. Briefly, despite much hard work, the situation is still messy, as synthetically summarized in a survey by Fiedler [11]: "An embracing systematic theory of homoclinic bifurcation in two parameters systems is not in sight. Rather, there appears to be hundreds of different cases which need to be analyzed separately."

Our aim here is to describe the striking organization around a remarkable parameter point, an organizational hub, discovered inside the chaotic phase of a circuit studied by Nishio et al. [12], our Fig. 1 below, containing a linear negative resistance and a nonlinear resistance formed by two diodes. We selected their nice circuit because it allows spirals to be measured experimentally. In addition, uncluttered by superfluous variables and parameters, their setup produces arguably the simplest possible normal form to experimentally observe spirals and hubs. We remark, however, that hubs and spirals are generic features because we also observed them in other familiar systems such as Rössler equations, in variations of Chua's circuit, and in some chemical and biological oscillators. As is clear from Fig. 2, the chaotic phase of the circuit contains infinite hierarchies of nested spirals which, altogether, compose a remarkably structured onionlike organization. In phase space, each individual spiral is characterized by a specific regular oscillation of a certain period which increases continuously beyond any bound when control parameters are suitably tuned along the spiral, towards the common central focus. Before commenting further on Fig. 2, we first explain how it was obtained.

The circuit in Fig. 1 defines an autonomous flow [12]:

$$
\frac{d x}{d t}=\alpha x+z, \quad \frac{d y}{d t}=z-f(y), \quad \frac{d z}{d t}=-x-\beta y .
$$

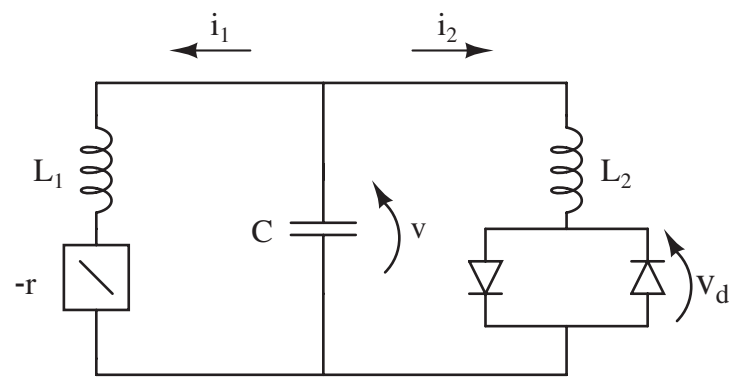

FIG. 1. The symmetric electronic circuit which displays the infinite hierarchy of nested spirals illustrated in Fig. 2. It involves negative linear and nonlinear resistive elements. 


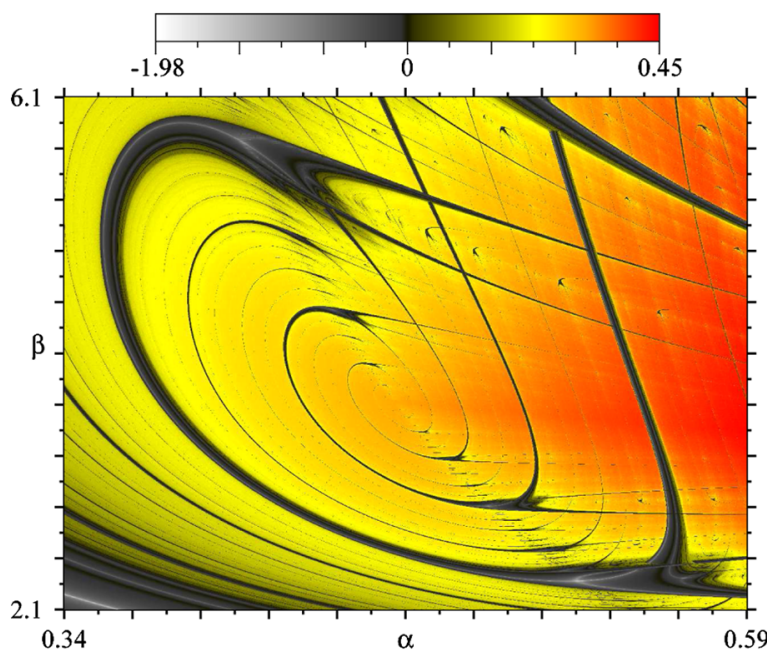

(a)

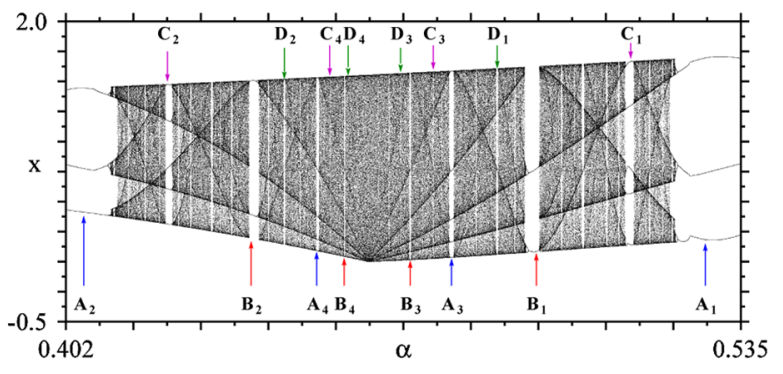

(c)

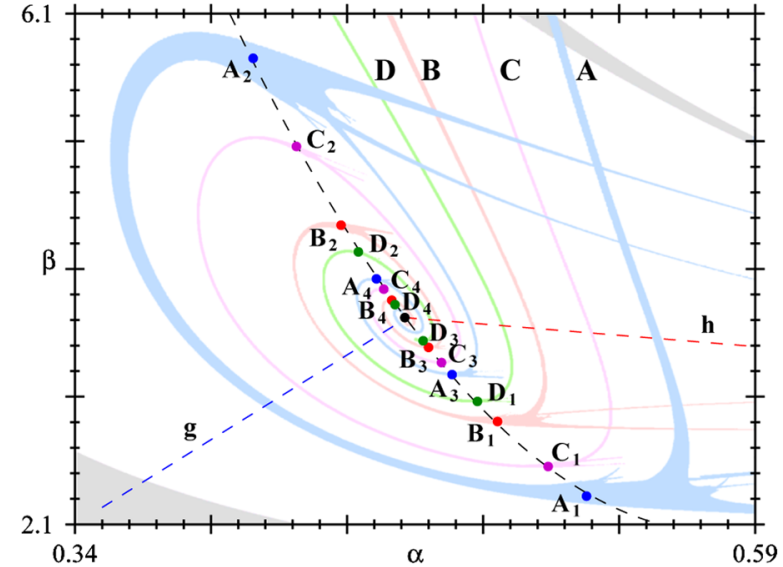

(b)

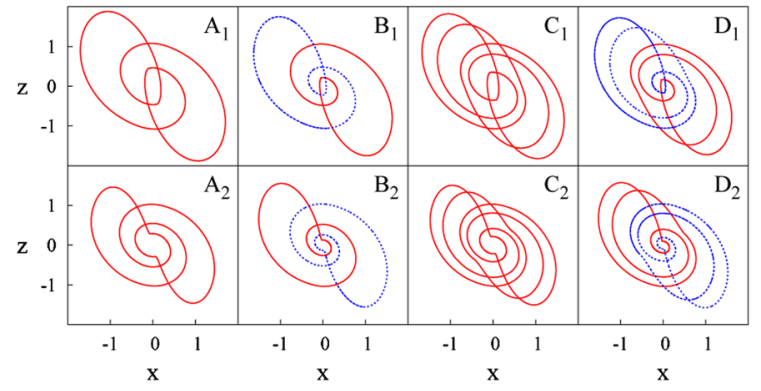

(d)

FIG. 2 (color online). (a) Phase diagram with an infinite hierarchy of nested spirals connected by the organizing hub at the focal point $\mathcal{F}$. The diagram displays the magnitude of the Lyapunov exponents. (b) Heads of the first few shrimps [13] $A_{i}, B_{i}, C_{i}, D_{i}$ emerging aligned along a parabolic arc, Eq. (4). See relevant data in Table I. Lines $h$ and $g$ are defined by Eqs. (5) and (6). (c) Bifurcation diagram along the parabolic arc through $\mathcal{F}$ and $A_{i}, B_{i}, C_{i}, D_{i}$. (d) Projections of the orbits in spirals $A, B, C, D$. In spirals $A$ and $C$ orbits are self-symmetric, while in $B$ and $D$ one finds coexistence of dual pairs in involution.

Here, $x$ and $y$ are scaled proxies of the currents $i_{1}$ and $i_{2}$ in Fig. $1, z$ is the scaled voltage drop $v$ across the capacitor $C$, and $\alpha$ and $\beta$ are free control parameters related with reactive elements in the circuit: $\alpha \equiv r \sqrt{C / L_{1}}$ and $\beta \equiv$ $L_{1} / L_{2}$. The piecewise linear resistance is

$$
f(y)=\frac{\gamma}{2}\left(\left|y+\frac{1}{\gamma}\right|-\left|y-\frac{1}{\gamma}\right|\right)
$$

where, following Nishio et al. [12], we fix $\gamma=470$, although our main result, Fig. 2(a), remains essentially unchanged in the wide interval $40 \leq \gamma \leq 1000$.

Figure 2(a) displays a phase diagram for the resistive circuit obtained by plotting Lyapunov exponents on a $1200 \times 900$ grid of equally spaced points. Equations (1) were integrated with a fixed-step $(h=0.005)$ fourth-order Runge-Kutta scheme. The first $35 \times 10^{3}$ steps were discarded, the subsequent $700 \times 10^{3}$ steps were used to compute the Lyapunov spectra. As known, negative exponents characterize periodic solutions while positive exponents are signatures of chaotic oscillations.
The phase diagram in Fig. 2(a) has two remarkable features: first, it contains an infinite nesting of spirals corresponding to periodic solutions; second, there is a distinctive focal point where all spirals originate or terminate and which organizes the dynamics in a wide portion of the parameter space around it. The focal point was numerically estimated to be roughly at

$$
\mathcal{F}=\left(\alpha_{f}, \beta_{f}\right)=(0.4612 \ldots, 3.7191 \ldots) .
$$

Individual spirals are characterized by specific families of periodic oscillations embedded in the chaotic phase. Spirals are formed by suitably "gluing" together leg to leg the characteristic four-legged dark domains called shrimps in Ref. [13] and which exist abundantly both in maps and flows [14]. Spirals and the spiral nesting are truly codimension-two phenomena: they may be only fully unfolded by tuning at least two parameters simultaneously.

Figure 2(b) shows how the four largest spirals, labeled $A$, $B, C, D$, coil up around $\mathcal{F}$. The doubly superstable points [15] defining shrimp heads were used to label the successive shrimps forming each spiral. Thus, circling clockwise 

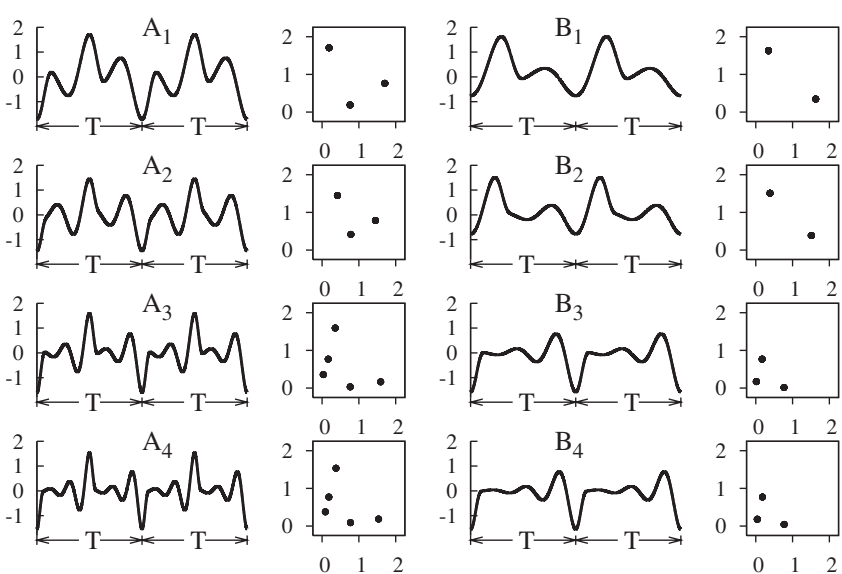
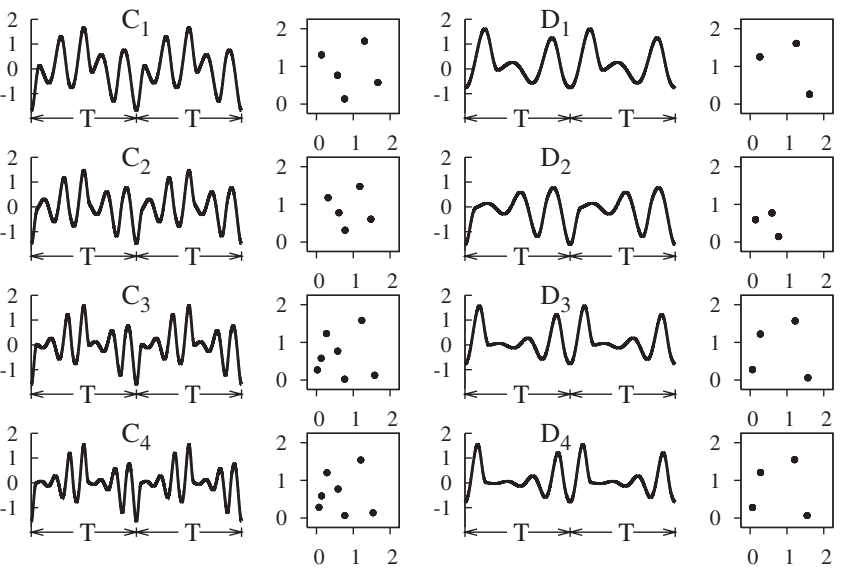

FIG. 3. Waveforms of the current $x(t)$ along spirals $A, B, C, D$ when moving towards the focal point $\mathcal{F}$. Return maps of the maxima $x_{t} \times x_{t+1}$ give the number of peaks. The number of peaks of $x(t)$ increases out of phase with respect to those of $y(t)$ and $z(t)$, as shown in Table I. Signals in self-symmetric spirals have much richer waveforms.

along spiral $A$ one meets successively the heads of shrimps $A_{1}, A_{2}$, etc. As is clear from the figure, the heads $A_{i}, B_{i}, C_{i}$, $D_{i}$, and $\mathcal{F}$ lie all along a distinctive direction, a parabolic arc, represented by a vertically slanted dashed curve in the figure. By fitting the shrimp heads of the four spirals $A_{i}, B_{i}$, $C_{i}, D_{i}$ for $i=1,2,3,4$ we found an approximate equation for this arc:

$$
\beta=124.5875 \alpha^{2}-143.7802 \alpha+43.5301 .
$$

Figure 2(b) contains two distinctive directions:

$$
\begin{aligned}
& \text { line } h: \quad \beta=-1.7604 \alpha+4.531, \\
& \text { line } g: \quad \beta=13.3912 \alpha-2.4569 .
\end{aligned}
$$

Line $h$, the homoclinic line, defines a unique direction in the phase diagram where all major shrimp legs not coiling up around $\mathcal{F}$ accumulate. In contrast, line $g$ marks a "generic" line on the left side of the parabolic arc. For any $g$ line on this side of the arc one finds the simplest possible sequence of bifurcations when moving to or from the focal point $\mathcal{F}$. Such bifurcation sequences remain invariant along any equivalent line passing through $\mathcal{F}$ and remaining on the left side of the parabolic arc.

The bifurcation diagram in Fig. 2(c) was obtained by simultaneously tuning $\alpha$ and $\beta$ along Eq. (4). It sheds light about the origin of the so-called periodic-chaotic sequences, also known as "period-adding" sequences [16-19]. Many such sequences reflect information gained from restricted one-parameter slices of codimension-two spiral nestings. A nice feature of Eqs. (1) is that they are invariant under the involution $(x, y, z) \mapsto(-x,-y,-z)$. This implies that every solution has always a "symmetric dual," which may be either self-symmetric or not; i.e., under the involution, orbital points will transform either to the same or to a distinct orbit. Both types of solutions are illustrated in Fig. 2(d).
The evolution of the current $x(t)$ along the four largest spirals is shown in Fig. 3 while Table I collects location, period, and number of peaks $p_{x}, p_{y}, p_{z}$ of the currents and voltage for the first few heads $A_{i}$ and $B_{i}$. Curiously, the number of peaks changes out of phase along the spirals, but always in a systematic way. A similar out-of-phase change occurs for $C$ and $D$ but starting from $\left(p_{x}, p_{y}, p_{z}\right)=(5,5,5)$ and $(3,3,3)$, respectively.

Figure 4 illustrates typical $z=0$ basins observed for spirals with nonself-symmetric dual attractors. As it is easy to realize, the strong "fractalization" of the basin boundaries poses great difficulties for predicting the behavior of a system as one moves towards the focal point, reminiscent of the difficulties familiar from Wada basins [20]. The basin $B_{4}$ was computed with $h=0.001$, the others with $h=0.005$. The ability to resolve basin structures when approaching $\mathcal{F}$ may certainly be used for benchmarking both experiments and numerical computations. For, experiments are strongly limited by noise of various kinds, while numerical work is limited by the accuracy of both computers and algorithms.

TABLE I. The number of peaks $p_{x}, p_{y}, p_{z}$ of currents $x(t), y(t)$ and voltage $z(t)$ evolve distinctly but regularly along the two types of spiral, self-symmetric or not. $T$ is the period of the oscillations while $(\alpha, \beta)$ locates the doubly superstable "centers" of the shrimps $[13,15]$ along spirals $A_{i}$ and $B_{i}$.

\begin{tabular}{ccccccc}
\hline \hline & $\alpha$ & $\beta$ & $T$ & $p_{x}$ & $p_{y}$ & $p_{z}$ \\
\hline$A_{1}$ & 0.52800 & 2.32100 & 15.965 & 3 & 3 & 3 \\
$A_{2}$ & 0.40550 & 5.75000 & 21.030 & 3 & 5 & 5 \\
$A_{3}$ & 0.47860 & 3.27290 & 28.355 & 5 & 5 & 5 \\
$A_{4}$ & 0.45082 & 4.02240 & 34.455 & 5 & 7 & 7 \\
$B_{1}$ & 0.49530 & 2.90520 & 11.040 & 2 & 2 & 2 \\
$B_{2}$ & 0.43780 & 4.44300 & 13.915 & 2 & 3 & 3 \\
$B_{3}$ & 0.46998 & 3.48562 & 17.360 & 3 & 3 & 3 \\
$B_{4}$ & 0.45644 & 3.85528 & 20.495 & 3 & 4 & 4 \\
\hline \hline
\end{tabular}



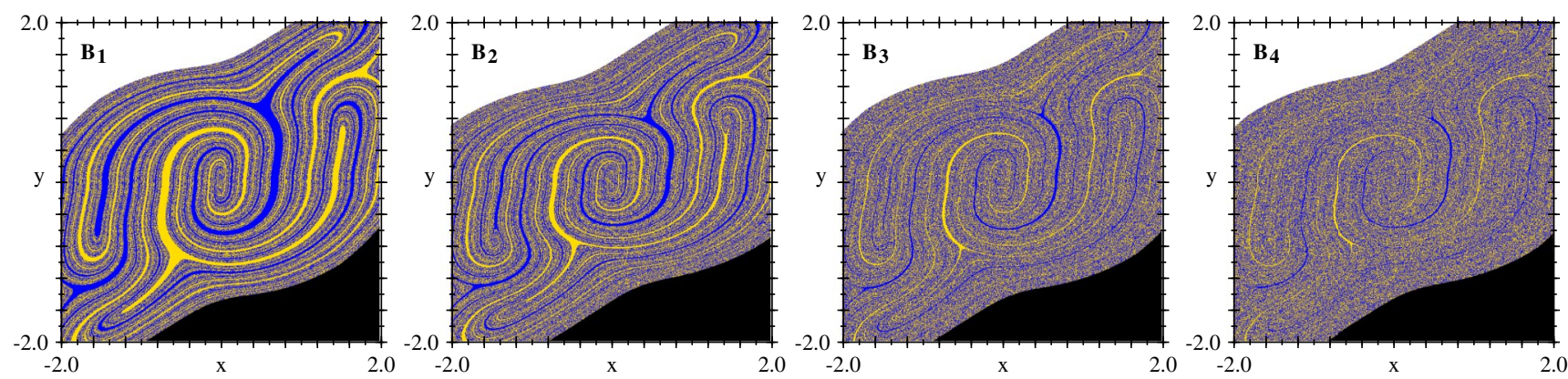

FIG. 4 (color online). Severe "fractalization" of the basins of attraction of nonself-symmetric dual attractors in spiral $B$ observed when marching towards $\mathcal{F}$. Black and white are basins of $\pm \infty$. The other two colors are the basins of the dual pair.

Finally, we mention that it is very tempting to associate spiral nestings with the much studied homoclinic orbits. However, numerical work shows spirals not to exist in some flows which are textbook examples of the Shilnikov setup. We described the unfolding of an infinite sequence of spirals in the vicinity of the numerically found focal hub. We believe our investigation to be accurate albeit not rigorous, and remark that we are not aware of any theory to predict and locate hubs. The parameter organization around hubs, in particular the regular spiral nesting, sheds new light on matters which seemed already well explored. We observed hubs and spirals in a broad spectrum of oscillators such as the Rössler equations, in variations of Chua's circuit, in certain chemical and biological oscillators and, therefore, expect them to be of importance in several fields, beyond the electronic circuit used as an illustrative example here. A key open question now is to investigate what sort of dynamical phenomena lead to hubs and spirals, the eventual role of homoclinic orbits in their genesis, and the mechanisms inducing periodicity transitions along and among spirals.

J. A. C. G. thanks F. T. Arecchi, E. Arimondo, E. Doedel, W. Govaerts, M. J. B. Hauser, B. Krauskopf, M. J. Ogorzalek, H. Osinga, C.L. Pando, A. Pisarchik, A. Politi, R. Roy, K. Showalter, and A. Vanderbauwhede for enlightening comments mostly during a fruitful Workshop in Nonlinear Dynamics and Complexity, Puebla, Mexico. The authors acknowledge support from CNPq, Brazil, and the AFOSR, Grant No. FA9550-07-1-0102.

[1] Handbook of Chaos Control, edited by E. Schöll and H. G. Schuster (Wiley-VCH, Weinheim, 2007).

[2] J. Argyris, G. Faust, and M. Haase, Die Erforschung des Chaos, Zweite Auflage (Springer, Berlin, 2008), 2nd ed.

[3] M.J. Ogorzalek, Chaos and Complexity in Nonlinear Electronic Circuits (World Scientific, Singapore, 1997).

[4] Digital Communications Using Chaos and Nonlinear Dynamics, edited by L.E. Larson, J.-M. Liu, and L.S. Tsimrig (Springer, New York, 2006).

[5] R. J. Field and L. Györgyi, Chaos in Chemistry and Biochemistry (World Scientific, Singapore, 1993).
[6] For a tutorial, see C. P. Silva, IEEE Trans. Circuit Syst. 40, 675 (1993); see also J. D. Meiss, Differential Dynamical Systems (SIAM, Philadelphia, 2007).

[7] S. Wiggins, Global Bifurcations and Chaos (Springer, New York, 1988).

[8] Y. A. Kusnetzov, Elements of Applied Bifurcation Theory (Springer, New York, 1998).

[9] P. Glendinning, Stability, Instability and Chaos (Cambridge University Press, Cambridge, England, 1994); P. Glendinning and C. Sparrow, J. Stat. Phys. 35, 645 (1984).

[10] G. Nicolis, Introduction to Nonlinear Science (Cambridge University Press, Cambridge, England, 1995); P. Gaspard, R. Kapral, and G. Nicolis, J. Stat. Phys. 35, 697 (1984).

[11] B. Fiedler, in Dynamics of Nonlinear Waves in Dissipative Systems: Reduction, Bifurcation and Stability, edited by G. Dangelmayr, B. Fiedler, K. Kirchgässner, and A. Mielke (Longman, Essex, 1996), Chap. 2.

[12] Y. Nishio, N. Inaba, S. Mori, and T. Saito, IEEE Trans. Circuit Syst. 37, 473 (1990).

[13] J. A. C. Gallas, Phys. Rev. Lett. 70, 2714 (1993); Physica (Amsterdam) 89D, 71 (1995); Physica (Amsterdam) 202A, 196 (1994); Appl. Phys. B 60, S-203 (1995).The previous two references introduce the canonical shrimp generator: $x_{t+1}=a-\left(b-x_{t}^{2}\right)^{2}$; see also Hunt et al., Physica (Amsterdam) 129D, 35 (1999).

[14] C. Bonatto, J. C. Garreau, and J. A. C. Gallas, Phys. Rev. Lett. 95, 143905 (2005); C. Bonatto and J. A. C. Gallas, Phys. Rev. E 75, 055204(R) (2007); Phil. Trans. R. Soc. A 366, 505 (2008); C. Bonatto, J. A. C. Gallas, and Y. Ueda, Phys. Rev. E 77, 026217 (2008).

[15] Although working with flows, for lack of a better name we keep using "doubly superstable points" here.

[16] F. Argoul et al., J. Chim. Phys. 84, 1367 (1987).

[17] D. Hennequin et al., Phys. Rev. A 37, 2243 (1988); D. Dangoisse et al., Europhys. Lett. 6, 335 (1988); F. T. Arecchi et al., Europhys. Lett. 6, 677 (1988); A. Zeni et al., Phys. Lett. A 172, 247 (1993).

[18] T. Braun, J. A. Lisboa, and J. A. C. Gallas, Phys. Rev. Lett. 68, 2770 (1992).

[19] A. Sensse, M. J. B. Hauser, and M. Eiswirth, J. Chem. Phys. 125, 014901 (2006).

[20] S. Takesue and K. Kaneko, Prog. Theor. Phys. 71, 35 (1984); H. E. Nusse and J. A. Yorke, Science 271, 1376 (1996); J. Aguirre and M. Sanjuan, Physica (Amsterdam) 171D, 41 (2002); Z. Hai-Lin et al., Chinese Phys. B 17, 117 (2008). 